\title{
Electronic nose and SPME techniques to monitor phenanthrene biodegradation in soil
}

\author{
Fabrizio De Cesare ${ }^{\mathrm{a}}$, Simone Pantalei ${ }^{\mathrm{b}}$, Emiliano Zampetti $^{\mathrm{b}}$, \\ Antonella Macagnano,
}

\author{
${ }^{a}$ Department of Agrobiology and Agrochemistry, University of Tuscia, \\ Via S. Camillo De Lellis, 01100 Viterbo, Italy \\ ${ }^{\mathrm{b}}$ Microelectronics and Microsystems Institute, National Research Council, \\ Via Fosso del Cavaliere 100, 00133 Rome, Italy
}

Available online 15 December 2007

\begin{abstract}
Phenanthrene is a hydrophobic organic pollutant, composed of three-fused benzene rings belonging to polycyclic aromatic hydrocarbons (PAHs). PAHs are produced by incomplete combustion of organic matter due to natural events, i.e. fires, and anthropogenic actions, i.e. C-containing fuels combustion. The presence of PAHs in contaminated soils is usually detected by classical extraction techniques, such as solvent extractions through Soxhlet extractor. In this work two recently developed techniques (solid phase microextraction-SPME and electronic nose-EN) able to analyse the headspace of solid or liquid samples were used to monitor the possible degradation of phenanthrene in an artificially contaminated soil. The analysis by SPME showed a drastic decrease of phenanthrene content after 30 days of incubation (-92\%) and different treatments with nutrient solutions and/or surfactant improve this rate up to 97\%. Differently, the analysis of soil headspaces by EN, processed by principal component analysis (PCA), showed that contaminated and uncontaminated soil samples (controls) might be distinguished on a temporal scale. Furthermore, PCA showed that phenanthrene-contaminated soil samples produced chemical images, which were delayed relative to controls at the same period of incubation. The application of partial least square-discriminant analysis (PLS DA) to chromatograms obtained by SPME pointed out the presence, in the headspace of phenanthrene-treated soils, of a series of possible indicators involved in phenanthrene degradation, which were completely absent in relative unpolluted controls. Results suggest that the two techniques do not necessarily represent mutually exclusive alternatives, but giving different information, they may be considered as complementary.
\end{abstract}

Keywords: Sensors; Electronic nose; Solid-phase microextraction; Soil bioremediation; Environmental applications 